18

\title{
Фотодинамика нелинейных эффектов при воздействии пикосекундного лазерного излучения на коллоидные растворы квантовых точек CdSe/ZnS*
}

\author{
(C) В.В. Данилов ${ }^{1,2}$, А.С. Кулагина ${ }^{3,4}$, Н.В. Сибирев ${ }^{4}$, А.И. Хребтов ${ }^{3}$, В.Б. Шилов ${ }^{2}$ \\ ${ }^{1}$ Петербургский государственный университет путей сообщения императора Александра I, \\ 190031 Санкт-Петербург, Россия \\ ${ }^{2}$ Государственный оптический институт им. С.И. Вавилова, \\ 199053 Санкт-Петербург, Россия \\ ${ }^{3}$ Санкт-Петербургский национальный исследовательский Академический университет РАН, \\ 194021 Санкт-Петербург, Россия \\ ${ }^{4}$ Университет ИТМО, \\ 197101 Санкт-Петербург, Россия \\ e-mail: vdanilov039@gmail.com
}

Поступила в редакцию 06.07.2018 г.

Рассмотрено влияние интенсивности воздействующего излучения на фотодинамику нелинейных процессов в коллоидных растворах квантовых точек $\mathrm{CdSe} / \mathrm{ZnS}$. Экспериментально исследовано и установлено различие нелинейных процессов, происходящих в течение времени непосредственного воздействия лазерного импульса и возникающих на стадии последействия. Исследование кинетики люминесценции в течение лазерного воздействия показало, что увеличение интенсивности приводит не только к эффекту оптического ограничения, но и к возникновению эффекта сверхизлучения для квантовых точек разных диаметров. На стадии последействия с помощью метода накачки-зондирования (pump-probe) обнаружен эффект ,динамического затвора“, обусловленного конкуренцией двух процессов: релаксацией через уровни размерного квантования и „бесфононной“ релаксацией через поверхностные или темные состояния. Математическая модель дезактивации высоковозбужденных состояний, записанная в форме уравнений Рикатти, показала хорошее совпадение с экспериментом.

DOI: $10.21883 /$ OS.2018.11.46831.192-18

В 70-е годы прошлого столетия исследование спонтанного излучения (люминесценции) привело к открытию новых оптических эффектов. Одно из них, коллективное спонтанное излучение многоатомной системы, или сверхизлучение, было теоретически предсказано Р. Дикке (R.H. Dicke) в 1954 г. [1]. В 1973 г. В. Данилов и Ю. Мазуренко при исследовании антистоксовой люминесценции растворов красителей при мощном лазерном возбуждении обнаружили, что с увеличением интенсивности возбуждения эффект светового тушения сменялся ростом квантового выхода люминесценции [2], связав это с эффектом Дикке. Однако дальнейшие исследования В. Смирнова и В. Шилова [3] привели к выводу о самостоятельной природе эффекта и возникновению теории коллективной фотоиндуцированной люминесценции (ФИЛ), объясняющей его происхождение [4]. Согласно представлениям авторов, коллективная фотоиндуцированная люминесценция является универсальным эффектом, проявляющимся при высоких концентрациях красителей и относительно интенсивном возбуждении как в стоксовой, так и в антистоксовой областях спектра. ФИЛ (как и эффект Дикке) на начальной стадии развивается экспоненциально в объемах, определяемых шириной

* International Conference „PCNSPA 2018 - Photonic Colloidal Nanostructures: Synthesis, Properties, and Applications“, Saint Petersburg, Russia, June 4-8, 2018. однородных спектров молекул [5], и может происходить только в течение времени сохранения фазовой памяти, пока молекулярная система находится в когерентном состоянии. Таким образом, его длительность не может превышать длительность возбуждающего импульса. Однако ФИЛ в отличие от эффекта Дикке не приводит к заселению возбужденных состояний молекул (в этом смысле ФИЛ представляет собой комбинационное рассеяние) и конкурирует с процессами обычного тушения, имеющими диффузионную природу, что существенно повышает квантовый выход люминесценции. Существование ФИЛ было подтверждено в последующих работах, где предметом исследования являлись растворы красителей и пары металлов [4-6]. Определенное сходство спектрального поведения растворов красителей и коллоидных растворов квантовых точек (КТ) поставило вопрос о возможности проявления ФИЛ или эффекта Дикке в растворах полупроводниковых наночастиц. Заметим, что в настоящее время экспериментальное проявление суперлюминесценции в наноструктурах является темой немалого количества публикаций, например [7-9].

Само сокращение длительности излучения принципиально меняет фотодинамику (комплекс процессов преобразования энергии света в произвольной среде с поглощением, см. подробнее [6]) ограничения в нелинейной среде [9-11]. Известно, что увеличение интен- 
сивности излучения (при переходе от наносекундного воздействия к пикосекундному) приводит к росту в квантовых точках (КТ) числа возбужденных свободных носителей, которые поглощают энергию излучения. Нами была поставлена задача, рассмотреть фотодинамику возбуждения в течение и после лазерного воздействия в растворах КТ. В процессе непосредственного воздействия наиболее простым и достаточно чувствительным методом является исследование зависимости амплитуды люминесценции от интенсивности возбуждающего излучения, которая позволяет выявить наличие нелинейностей и диапазоны энергий, в которых они доминируют. Как мы уже отмечали, индикатором спектрально кинетических изменений, происходящих в исследуемой среде при вариации интенсивности лазерного излучения, является эффект оптического ограничения [12-15]. Информацию о процессах, происходящих на стадии последействия, можно получить с помощью метода накачкизондирования (или pump-probe) [16,17]. В этом случае особую роль при интерпретации процессов приобретает сопоставление результатов нелинейного пропускания и кинетики.

\section{Материалы и оборудование}

В качестве объектов исследования использовались полупроводниковые КТ $\mathrm{CdSe} / \mathrm{ZnS}$ с разным диаметром ядра $(\mathrm{CdSe}): 2.5 \mathrm{~nm}\left(5 \cdot 10^{6} \mathrm{M}\right)$ и $4.9 \mathrm{~nm}\left(4.5 \cdot 10^{7} \mathrm{M}\right)$, стабилизированные молекулами ТОФО (триоктилфосфиноксид). Все измерения проводились для растворов в толуоле. Наночастицы синтезированы в Институте физико-химических проблем БГУ.

В экспериментах по лазерному ограничению и кинетике нелинейного отклика на стадии последействия в растворах КТ (большего диаметра) источником излучения служил $\mathrm{YAG}: \mathrm{Nd}^{2+}$-лазер с пассивной модуляцией добротности. Исходное излучение $\lambda=1064 \mathrm{~nm}, \tau=7 \mathrm{~ns}$ фокусировалось в кювету с $\mathrm{CCl}_{4}$ и сжималось посредством ВРМБ до длительности 400 ps. С помощью кристалла КТР происходило удвоение частоты, после чего лазерный импульс преобразовывался с помощью ВКР в кювете с $\mathrm{CF}_{4}$, на выходе длина волны составляла $560 \mathrm{~nm}$, а длительность импульса 20 ps. Расходимость излучения составляла около $1.5 \mathrm{mrad}$, диаметр пучка - около $5 \mathrm{~mm}$. С помощью оптического клина лазерный импульс делился на два, после ослабления накачки с помощью нейтральных фильтров до величины, соответствующей нелинейному снижению пропускания образца в 2 раза, соотношение интенсивностей зондирующего (или пробного) импульса к накачке составило 1:2. Временная задержка между зондирующим импульсом и накачкой регулировалась с помощью линии задержки с ценой деления $1 \mathrm{~mm}$. Максимальная задержка составляла около 600 ps. Излучение пробного пучка вводилось в кювету с исследуемым раствором под углом $7^{\circ}$ к накачке и на выходе регистрировалось фотодиодным приемником.
В области перекрытия пучков диаметр пятна накачки составлял около $150 \mu \mathrm{m}$, пробного - около $60 \mu \mathrm{m}$. Экспериментальные результаты впервые обсуждались в работе [18], там же представлена оптическая схема. Динамика люминесценции КТ (меньшего диаметра) при интенсивном возбуждении исследовалась с использованием щелевой камеры Hamamatsu с временным разрешением до $10 \mathrm{ps}$. Исследование растворов в кварцевых кюветах $1 \mathrm{~cm}$ проводилось в квазипараллельном пучке при следующих параметрах излучения: $\lambda=532 \mathrm{~nm} \mathrm{и}$ $\tau_{\text {pulse }}=400$ ps. Сигнал люминесценции собирался короткофокусной линзой под углом $90^{\circ}$ к накачке. По отношению к полосе поглощения возбуждение люминесценции имело антистоксов характер.

\section{Результаты и обсуждение}

На рис. 1 приведены экспериментальные результаты измерений кинетики люминесценции коллоидных растворов КТ при интенсивном лазерном воздействии. Видно, что при увеличении интенсивности возбуждения время затухания люминесценции резко сокращалось до величины порядка $0.35 \mathrm{~ns}$, сопоставимой с длительностью лазерного импульса; кроме того, амплитуда люминесценценции резко возросла. Возникновение такой „суперлюминесценции“ позволяет предположить процесс излучательной рекомбинации с участием поверхностных дефектов.

Уменьшение длительности флуоресценции находится в соответствии с концепцией ФИЛ аналогично эффекту, наблюдаемому в растворах красителей [6]. Эта флуоресценция обладает следующими признаками: во-первых, она возникает на фоне интенсивного светового тушения [19], во-вторых, имеет пороговый характер, в-третьих, длительность этой люминесценции при определенных уровнях возбуждения повторяет длительность

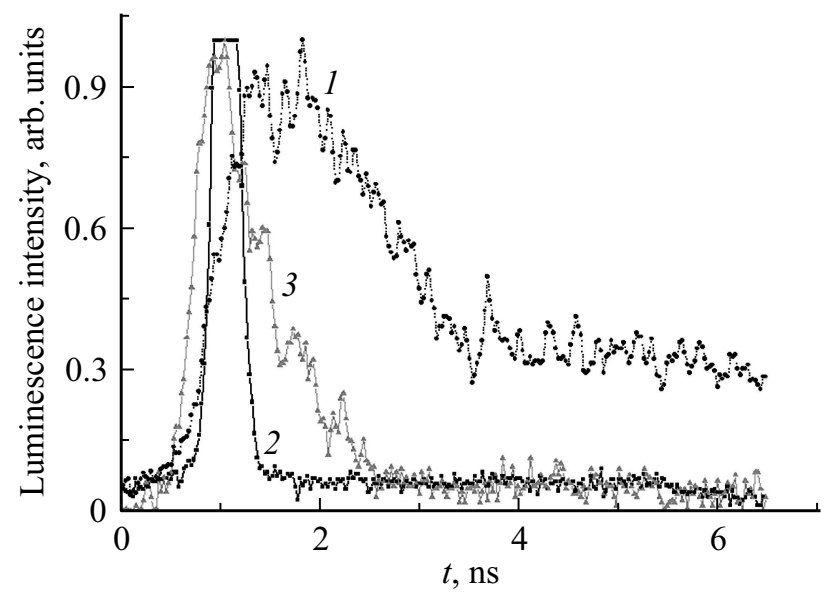

Рис. 1. Кинетика люминесценции $\mathrm{CdSe} \mathrm{ZnS}$ КТ диаметром $2.5 \mathrm{~nm}$ при интенсивностях возбуждения 2 (кривая 1) и $15 \mathrm{GW} / \mathrm{cm}^{2}(2) ; 3$ - уровень шума люминесценции пустой кюветы при $2 \mathrm{GW} / \mathrm{cm}^{2}$. 
возбуждающего импульса. Согласно теории ФИЛ, в стоксовой области при больших энергиях импульса накачки происходит насыщение заселенности возбужденного состояния $S_{1}$, при котором уровень ФИЛ, определяемый величиной $\left(1-\alpha S_{1}\right)$ (при $S_{1} \rightarrow 1$ переход является насыщенным), достаточно высок. Здесь $\alpha-$ сечение поглощения. Если предположить, что потери излучения определяются фотоиндуцированной флуоресценцией, то ослабление пучка $\xi$ вдоль оси распространения $x$ будет определяться уравнением $d \xi / d x=-0.1 \alpha N(N-$ концентрация КТ), т.е. пучок будет активно затухать на небольших расстояниях. При возбуждении в антистоксовой области значения сечения поглощения меньше сечения вынужденного излучения, поэтому насыщение заселенности возбужденного состояния происходит при малых величинах возбуждающего сигнала, что приводит к просветлению раствора с увеличением энергии импульса. При этом в обоих случаях надо учитывать конкуренцию оже-процессов, ведущих, в частности, к заселению высших возбужденных состояний. Однако дальнейшее обсуждение результатов не позволяет полностью исключить в этом случае и механизм Дикке.

Кинетика последействия, исследованная с помощью метода накачки-зондирования, приведена на рис. 2, $a$. Отрицательная шкала времени введена условно, для сопоставления нулю полного пространственного и временного совпадения импульсов накачки и зондирующего. Стадия просветления и общая динамика нелинейного пропускания обсуждались нами ранее в [18]. Здесь подробнее остановимся на этапах ограничения и восстановления пропускания, соответствующих временной задержке от 150 до 400 ps. Одновременно высокие концентрации электронов проводимости и дырок возможны при интенсивном возбуждении полупроводника светом. При высоких уровнях возбуждения в поглощении доминируют оже-процессы [9]. Более того, в условиях интенсивного возбуждения оже-релаксация через состояние $1 S(e)$ означает (согласно $[10,20,21]$ ) заполнение более высоких энергетических уровней, что и приводит при моновоздействии к эффекту ограничения.

Особенностью наблюдаемой картины фотодинамики является появление „эффекта отложенного ограничения“ (рис. 2,a). Вначале наблюдается медленный рост заселенности поглощающих состояний, характерное время около 300 ps, а также следующая за ним чрезвычайно короткая стадия восстановления исходного пропускания, характерное время менее 70 ps. Наличие медленной стадии перед быстрой заставляет предположить наличие нелинейных эффектов. Обычно такое короткое время востановления связывают с процессами дезактивации поверхностных возбужденных состояний, которые благодаря в том числе и оже-процессам образуются при возбуждении $[22,23]$. Однако наличие длительной предшествующей стадии (линейный участок на рис. 2,a) заставляет предположить существование своеобразного динамического затвора, препятствующего переходу носителей из $S_{1}$ в основное состояние. Схема на рис. $2, b$
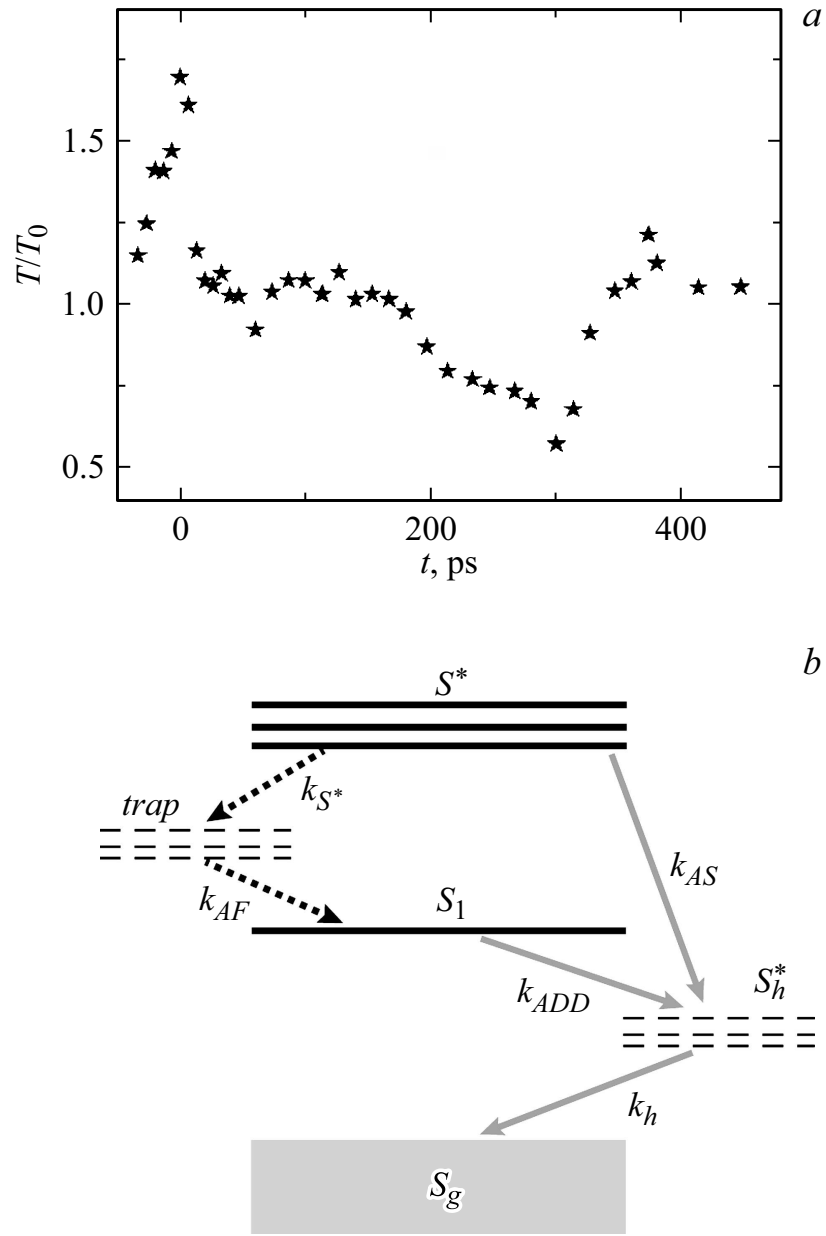

Pис. 2. (a) Кинетика оптического пропускания раствора КТ $\mathrm{CdSe} \mathrm{ZnS}$ диаметром $4.9 \mathrm{~nm}$. (b) Схематическое представление релаксации возбуждения,полученное на основе сопоставления экспериментальных данных и результатов расчета. $S^{*}-$ высшие возбуждённые состояния $(E \geq 2.45 \mathrm{eV}$ для исследуемых КТ); trap - объёмные ловушечные состояния; $S_{1}$ - нижнее возбуждённое состояние ( $E \approx 2.03 \mathrm{eV}$ для исследуемых КТ); $S_{h}^{*}$ - дырочные уровни; $S_{g}$ - основное состояние.

представляет возможные пути релаксации возбужденных носителей, исходно сосредоточенных на высших электронных состояниях $S^{*}$. При интерпретации результатов мы традиционно исходим из существования двух дырочных состояний $S_{h}\left(S_{1}\right.$ на рис. $\left.2, b\right)$ и $S_{h}^{*}$ [24-26], разделенных энергетическим барьером $\Delta E$ [24,25] (заметим, что состояние $S_{h}^{*}$ часто интерпретируется как темное состояние [21]). Скорее всего, дезактивация из нижнего возбужденного состояния $S_{1}$ и высоковозбужденных состояний $S^{*}$ предпочтительно идет через одно и то же состояние $S_{h}^{*}$. Пока в высоковозбужденных состояниях $S^{*}$ находится много носителей, релаксация из $S_{1}$ в состояние $S_{h}^{*}$ ограничена. В момент, когда высоковозбужденные состояния истощаются, скорость релаксации из состояния $S_{1}$ резко возрастает. С другой стороны, на медленной стадии заполненность состояния $S_{1}$ растет 


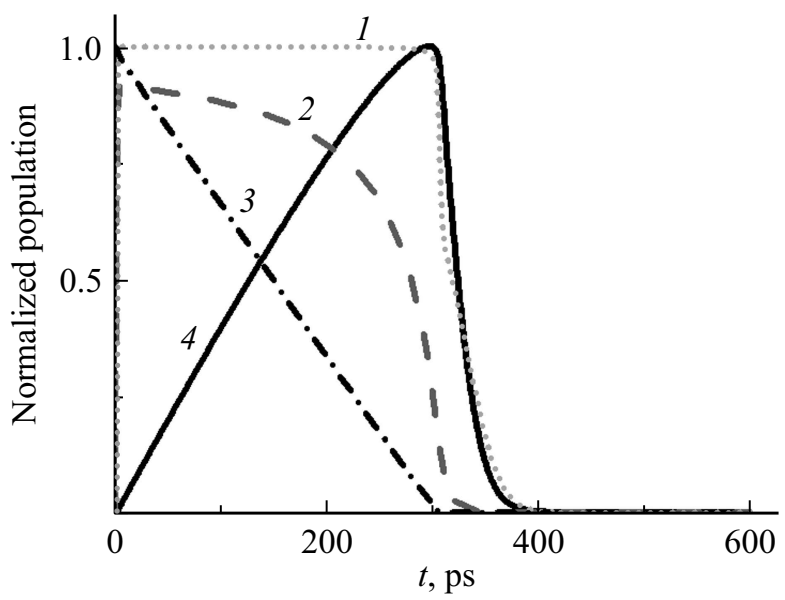

Рис. 3. Кинетическая диаграмма заполнения энергетических состояний: 1 соответствует уровням $S_{h}^{*}\left(\frac{S_{h}^{*}}{\tilde{S}_{h}^{*}}\right.$ по шкале $\left.y\right)$; 2 соответствует уровням $\operatorname{trap}\left(\frac{T}{T}\right) ; 3$ соответствует уровням $S^{*}$ $\left(\widetilde{S}^{*}\right) ; 4$ соответствует уровням $S_{1}\left(\frac{S_{1}}{\max _{t}\left(S_{1}\right)}\right)$.

почти линейно (рис. 3), что, по-видимому, означает, что дезактивация высоковозбужденных состояний в нижнее возбужденное состояние $S_{1}$ (так называемое „светлое“) происходит в два этапа: по уровням энергии размерного квантования и дальше через ловушечные уровни trap. При этом дезактивация самого состояния $S_{1}$ идет предпочтительно через состояние $S_{h}^{*}$. В то же время проявление динамического затвора свидетельствует о наличии безызлучательного канала релаксации в масштабах времени $1-10 \mathrm{ps}$, который доступен электронам и/или дыркам в условиях сильного конфаймента для коллоидных КТ [27]. Заметим, что авторы работы [28] предполагают, что поверхность КТ может быть неотьемлемой частью такого внутризонного релаксационного механизма.

Другими словами,анализ фотодинамики процесса говорит о существовании двух типов каналов дезактивации возбуждения, проходящих через „светлое“ состояние $S_{1}$ и в обход. Полная кинетическая схема процесса состоит из элементарных стадий, которые могут быть описаны системой дифференциальных уравнений типа Рикатти:

$$
\left\{\begin{array}{l}
\frac{d S^{*}}{d t}=-k_{S^{*}} S^{*}\left(1-\frac{T}{\widetilde{T}}\right)-k_{A S} S^{*}\left(1-\frac{S_{h}^{*}}{S_{h}^{*}}\right), \\
\frac{d T}{d t}=k_{S^{*}} S^{*}\left(1-\frac{T}{\widetilde{T}}\right)-k_{A F} T, \\
\frac{d S_{1}}{d t}=k_{A F} T-k_{A D D} S_{1}\left(1-\frac{S_{h}^{*}}{S_{h}^{*}}\right), \\
\frac{d S_{h}^{*}}{d t}=k_{A S} S^{*}\left(1-\frac{S_{h}^{*}}{S_{h}^{*}}\right)+k_{A D D} S_{1}\left(1-\frac{S_{h}^{*}}{S_{h}^{*}}\right)-k_{h} S_{h}^{*}
\end{array}\right.
$$

Здесь $S^{*}$ - суммарная заселенность верхних возбужденных уровней, $S_{1}$ - заселенность нижнего „светлого “ возбужденного уровня, $S_{h}^{*}-$ суммарная заселенность второго дырочного уровня, $T-$ заселенность ловушечных уровней trap. $\breve{T}$ и $\breve{S}_{h}^{*}-$ константы, характери- зующие предельную заполненность ловушек и состояния $S_{h}^{*}$ соответственно. $k_{S^{*}}$ - скорость релаксации их верхних возбужденных состояний $S^{*}$ на уровни trap; $k_{A F}$ - скорость перехода с уровней trap на нижнее возбужденное состояние $S_{1} ; k_{A S}-$ скорость релаксации на уровни $S_{h}^{*} ; k_{h}-$ скорость безызлучательной рекомбинации состояний trap в основное состояние $S_{g} ; k_{A D D}-$ скорость безызлучательной деактивации уровня $S_{1}$. Для корректного решения уравнений необходимо задать начальные условия. В нашем случае после импульса все уровни, кроме высоковозбужденных состояний, не заселены $S_{1}(t=0)=T(t=0)=S_{h}^{*}(t=0)=0$. А начальная заселенность высоковозбужденных состояний $\hat{S}^{*}$, $\left.S^{*}(t=0)=\hat{S}^{*}\right)$.

Из диаграммы на рис. 3 следует,что благодаря преимуществу в скорости канал безызлучательной дезактивации состояния $S_{1}$ оказывается заблокированным до момента полной релаксации уровней $S^{*}$. Накопление возбуждения на $S_{1}$, приводит к появлению эффекта „отложенного ограничения“. С окончанием дезактивации $S^{*}$ включается дезактивация $S_{1}$ через $S_{h}^{*}$, и система возвращается в основное состояние. Выполненные расчеты показывают, что теоретическая зависимость соответствует экспериментальным результатам при следующих значениях динамических констант $(k=1 / \tau)$ : $\tau_{S}^{*}=371 \mathrm{ps}, \tau_{A F}=8.23 \mathrm{ps}, k_{A S}=10^{13} \mathrm{~s}^{-1}, \tau_{h}=3.25 \mathrm{ps}$, $k_{A D D}=8.4 \cdot 10^{10} \mathrm{~s}^{-1}, \quad \breve{T}=0.002 \hat{S}^{*}, \quad \breve{S}_{h}^{*}=0.01 \hat{S}^{*} . \quad$ На рис. 4 представлено сравнение измеренной кинетики ограничения и решения уравнений Рикатти (1). Полученные теоретические значения релаксационных констант соответствуют также экспериментальным значениям,известным из литературы $[23,26]$. И то, что при этих значениях мы получили хорошее совпадение с экспериментом, говорит в пользу нашей интерпретации.

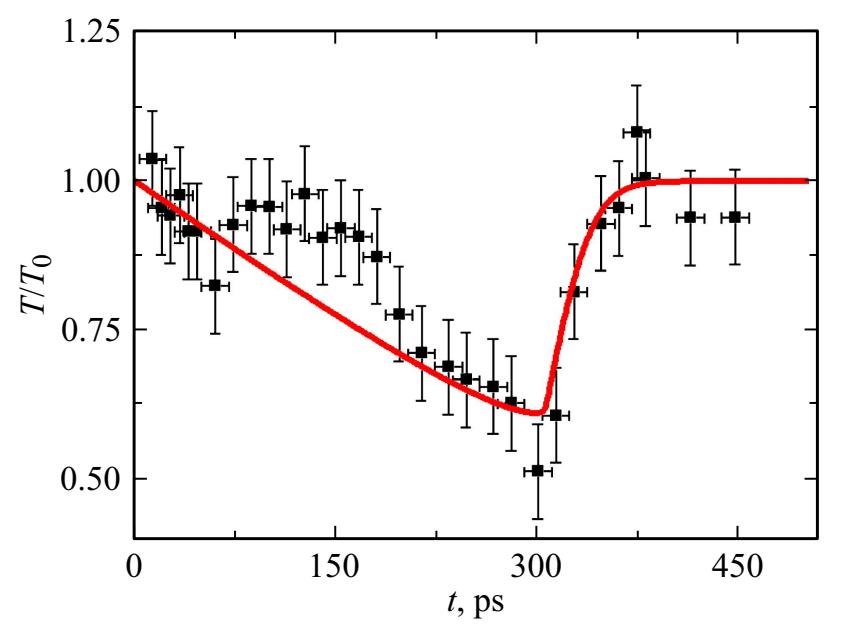

Рис. 4. Аппроксимация экспериментальных данных по кинетике ограничения (точки) решением системы (1). Разброс по вертикальной шкале соответствует рассчитанному стандартному отклонению; разброс по горизонтальной шкале показывает неопределённость положения точки на шкале времени, обусловленную длительностью лазерного импульса. 
Таким образом, возникающие при высоких плотностях воздействующего излучения заряженные состояния экситонов открывают дополнительный, связанный с ожепроцессом, путь заполнения высокоэнергетических состояний КТ фотовозбужденными носителями. Однако поскольку в полупроводниковых КТ энергетический зазор между энергетическими уровнями пространственного квантования заметно превышает энергию оптических фононов, то обратный процесс фононной релаксации затруднен. Возбужденные носители заряда КТ не могут попасть в основное состояние по уровням энергии размерного квантования и „вынуждены“ задерживаться на высокоэнергетических уровнях (так называемый эффект „бутылочного горла“) [22]. Тем не менее в ряде работ $[23,26]$ сообщается о существовании дополнительных каналов быстрой релаксации, снижающих влияние этого эффекта. Одним из них является релаксация с участием поверхностных дефектов. Например, избыточная энергия может теряться электроном при испускании акустических фононов в процессе туннелирования с КТ на дефект и обратно. В этом случае, скорости релаксации достигают при комнатных температурах $\sim 10^{12} \mathrm{~s}^{-1}[20]$. $\mathrm{B}$ нашей схеме по такому механизму работает канал I, т. е. изначально медленный процесс охлаждения электрона, пройдя через ловушку trap, ускоряется и приводит к накоплению возбуждения на долгоживущем $S_{1}$-состоянии. В то же время возникающие в оже-процессе дырки легко захватываются на поверхностные ловушки в точках пассивации поверхности органическими лигандами $[22,26]$. В ряде работ (например, [20-22]) показано, что при достаточно сильной электронно-дырочной ожесвязи становится эффективной передача избыточной энергии горячих электронов дыркам (так называемая бесфононная релаксация), которые быстро релаксируют в основное состояние. Поэтому дезактивация $S^{*}$ может происходить более эффективно по каналу II, связанному напрямую с релаксацией через состояния $S_{h}^{*}$, которые по определению являются дырочными. Мы не исключаем участия в этом процессе поверхностных состояний. Длительность такого процесса достигает долей пикосекунд, что можно объяснить высокой плотностью состояний валентной зоны и небольшим радиусом локализации дырок. Таким образом, безызлучательный распад возбужденных электронных состояний может быть быстрым при условии, что избыточная энергия электрона может быть эффективно перенесена на дырку [24]. В работе [10] оба эти процесса: замедление релаксации носителей по уровням энергии размерного квантования при большой концентрации возбужденных электроннодырочных пар и быстрая бесфононная релаксация энергии горячих электронов за счет передачи энергии быстро релаксирующим дыркам - наблюдались по отдельности при варьировании условиями эксперимента. В нашем случае оба конкурирующих процесса наблюдались одновременно. Отметим, что в общем случае эффект „бутылочного горла“, т.е. замедление релаксации из-за квантовых запретов, может нивелироваться процессом диполь-дипольного переноса возбуждения с КТ по механизму FRET на молекулы ТОФО или/и локальные связи $(\mathrm{C}-\mathrm{H})$ растворителя путем электрон-колебательного размена $[14,29,30]$.

Работа выполнена при поддержке гранта Министерства образования и науки Российской Федерации, проект 16.9791.2017/8.9.

\section{Список литературы}

[1] Dicke R.H. // Phys. Rev. 1954. V. 93. P. 99.

[2] Danilov V.V., Mazurenko Yu.T., Vorontzova S.I. // Opt. Commun. 1973. V. 9. N 3. P. 283.

[3] Ермолаева Г.М, Грегг Е.Г., Смирнов В.А, Шилов В.Б. // Опт. и спектр. 1998. Т. 84. № 3. С. 393.

[4] Смирнов В.А., Ермолаева Г.М., Шилов В.Б. // Опт. и спектр. 2002. Т. 92. № 6. С. 923.

[5] Данилов В.В., Смирнов В.А., Шилов В.Б. // Оптический журн. 2004. Т. 71. № 2. С. 73.

[6] Данилов В.В. Прикладная фотодинамика для инженеров / Учебное пособие. СПб: ФГБОУ ВО ПГУПС, 2016. 132 с.

[7] Воробьев Л.Е., Паневин В.Ю., Федосов Н.К., Фирсов Д.А., Шалыгин B.A., Hanna S., Seilmeier A., Moumanis Kh., Julien F., Жуков A.E., Устинов В.М. // ФТТ. 2004. Т. 46. № 1. C. 119.

[8] Scheibner M., Schmidt T., Worschech L., Forchel A., Bacher G., Passow T., Hommel D. // Nature Physics. 2007. V. 3. P. 106.

[9] Henry E., Dif A., Schmutz M., Legoff L., Amblard F., MarchiArtzner V., Artzner F. // Nano Lett. 2011. V. 11. N 12. P. 5443.

[10] Днепровский В.С., Добындэ И.И., Жуков Е.А., Санталов А.М. // ФТТ. 2007. Т. 49. № 4. С. 741.

[11] Dob̌̌ndă I.I. // Moldavian Journal of the Physical Sciences. 2007. V. 6. N 3. P. 373.

[12] Danilov V.V., Panfutova A.S., Khrebtov A.I., Ambrosini S., Videnichev D.A. // Optics Lett. 2012. V. 37. N 19. P. 3948.

[13] Данилов В.В., Панфутова А.С., Шилов В.Б., Белоусова И.М., Ермолаева Г.М., Хребтов А.И., Веденичев Д.А. // Химическая физика. 2015. Т. 34. № 8. С. 58.

[14] Bodunov E.N., Danilov V.V., Panfutova A.S., Simoes Gamboa A.L. // Annalen der Physik. 2016. V. 528. N 3-4. P. 272.

[15] Gryaznova M.V., Danilov V.V., Khapova O.V., Khrebtov A.I., Shakhverdov T.A. // Quant. Electron. 2004. V. 34. N 5. P. 407.

[16] Vivien L., Riehl D., Delaire J. // JOSA B. 2002. V. 19. N 2. P. 208.

[17] Van Dao L., Wen X.M., Do M.T.T., Hannafor P., Cho E.C., Cho Y.H., Huang Y.D. // J. Appl. Phys. 2005. V. 97. P. 013501.

[18] Кулагина А.С., Данилов В.В., Шилов В.Б., Григоренко К.М., Власов В.В., Ермолаева Г.М. // Опт. и спектр. 2017. T. 123. № 1. C. 152.

[19] Данилов В.В., Панфутова А.С., Шилов В.Б., Белоусова И.М., Ермолаева Г.М., Хребтов А.И. // Опт. и спектр. 2014. T. 116. № 6. С. 129.

[20] Федоров А.В., Рухленко И.Д., Баранов А.В., Кручинин С.Ю. Оптические свойства полупроводниковых квантовых точек. М.: Наука, 2011. 188 с.

[21] Dneprovskii V.S., Kanev A.R., Kozlova M.V., Smirnov A.M. // Proc. SPIE. 2014. V. 9136. P. 9136Y-1.

[22] Guyot-Sionnest P., Wehrenberg B., Yu D. // J. Chem. Phys. 2005. V. 123. P. 074709. 
[23] An J.M., Califano M., Franceschetti A., Zunger A. // J. Chem. Phys. 2008. V. 128. P. 164720.

[24] Efros Al.L., Rosen M., Kuno M., Nirmal M., Norris D.J., Bawendi M. // Phys. Rev. B. 1996. V. 54. P. 4843.

[25] Klimov V.I., Mikhailovsky A.A., Xu S., Malko A., Hollingsworth J.A., Leatherdale C.A., Wehrenberg B.L., Wang C., Guyot-Sionnest P. // J. Phys. Chem. B. 2002. V. 106. P. 10634.

[26] Woggon U., Giessen H., Gindele F., Wind O., Fluegel B., Peyghambarian N. // Phys. Rev. B. 1996. V. 54. P. 17681.

[27] Harbold J.M., Du H., Krauss T.D., Kyung-Sang Ch., Murray C.B., Wise F.W. // Phys. Rev. B. 2005. V. 272. P. 195312

[28] Blackburn J.L., Selmarten R.J., Ellingson R.J., Jones M., Micic O., Nozik A.J. // J. Phys. Chem. B. 2003. V. 107. P. 102.

[29] Данилов В.В., Хребтов А.И., Штром И.В., Цырлин Г.Э., Самсоненко Ю.Б. // Опт. и спектр. 2016. Т. 121. № 3. C. 405.

[30] Danilov V.V., Khrebtov A.I., Shtrom I.V., Cirlin G.E., Samsonenko Yu.B. // J. Nanomaterials. 2015. V. 2015. Article ID 713837 (4 pages). 